\title{
ON APPROXIMATION BY SHIFTS AND A THEOREM OF WIENER
}

\author{
BY
}

R. A. ZALIK

\begin{abstract}
We study the completeness in $L_{2}(R)$ of sequences of the form $\left\{f\left(c_{n}-t\right)\right\}$, where $\left\{c_{n}\right\}$ is a sequence of distinct real numbers. A Müntztype theorem is proved, valid for a large class of functions and, in particular, for $f(t)=\exp \left(-t^{2}\right)$.
\end{abstract}

Let $c, t, x$ and $y$ denote real numbers, and $z, w$ complex numbers, let $R$ stand for the set of real numbers, let $f$ be a function in $L_{2}(R)$, and let $T(f)$ denote the linear span of the family of functions of the form $f(t+c), c$ real. A classical theorem of $\mathrm{N}$. Wiener [1, p. 100, Theorem 12] asserts that the closure of $T(f)$ equals $L_{2}(R)$ if, and only if, the set of points at which the Fourier transform of $f$ vanishes has measure zero. The question thus naturally arises as to under what conditions a sequence of the form $\left\{f\left(t+c_{n}\right)\right\}$ will span $L_{2}(R)$. Our first result in this direction is the following:

THEOREM 1. Let $\left\{c_{n}\right\}$ be a sequence of distinct real numbers. Let $-\infty<a<$ $b<\infty$, and assume that $f$ belongs to $L_{2}(R)$ and is such that the sequence $\left\{f\left(c_{n}-t\right)\right\}$ spans $L_{2}(2 a, 2 b)$. Let $g$ be a function in $L_{1}(R)$ with support in $(a, b)$, and such that the set of points at which the Fourier transform of $g$ vanishes has measure zero. Then, if $h=f * g$ is the convolution product of $f$ and $g$, the sequence $\left\{h\left(c_{n}-t\right)\right\}$ spans $L_{2}(a, b)$.

In particular, we have the following:

CoRollary 1. Assume that the sequence $\left\{f\left(c_{n}-t\right)\right\}$ spans $L_{2}(R)$, that $g$ belongs to $L_{1}(R)$, and that the Fourier transform of $g$ is different from zero almost everywhere on $R$. Then, if $h=f * g$ is the convolution product of $f$ and $g$, also the sequence $\left\{h\left(c_{n}-t\right)\right\}$ spans $L_{2}(R)$.

Theorem 1 will be useful only if we can show there exists functions $f$ and sequences $\left\{c_{n}\right\}$ such that $\left\{f\left(c_{n}-t\right)\right\}$ spans $L_{2}$. This will follow from the next theorem, which is our principal one, and also from Theorem 4. Given a

Received by the editors July 10, 1977.

AMS (MOS) subject classifications (1970). Primary 41A30, 42A64, 30A62, 30A70; Secondary 42A68, 42A96, 44A35.

Key words and phrases. Approximation by sequences of shifts in $L_{2}(R)$, entire functions of order 2, Fourier inversion. 
function $f$, by $F$ we shall denote its (exponential) Fourier transform. Given a sequence $\left\{c_{n}\right\}$, the expression

$$
\sum^{\prime}\left|c_{n}\right|^{-(2+e)} \quad(\varepsilon \geqslant 0)
$$

will denote the sum of all terms of the form indicated, with nonvanishing denominator. Let $\left\{c_{n}\right\}$ be a given sequence of distinct real numbers, and for this sequence let $S(\varepsilon)$ denote the series (1), where $\varepsilon$ is an arbitrary nonnegative real number. With this notation we have

THEOREM 2. Let $f$ be a function in $L_{2}(R)$ such that $F(x) \neq 0$ for all real $x$, and consider the following conditions:

(i) There is a nonzero real number $a$, such that

$$
F(x)=O\left[\exp \left(-a^{2} x^{2}\right)\right], \quad|x| \rightarrow \infty .
$$

(ii) There is a nonzero real number $b$, such that $\left[\exp \left(-b^{2} x^{2}\right)\right] / F(x)$ is in $L_{2}(R)$.

Then, if (i) holds, the divergence of $S(\varepsilon)$ for some $\varepsilon>0$ suffices for the sequence $\left\{f\left(c_{n}-t\right)\right\}$ to span $L_{2}(R)$, whereas if (ii) holds, the divergence of $S(0)$ is necessary for this sequence to span $L_{2}(R){ }^{1}$

EXAMPLE. The function $f(t)=\exp \left(-t^{2}\right)$ satisfies conditions (i) and (ii) of Theorem 2.

Let $f_{n}(t)=f\left(c_{n}-t\right)$, and let $F_{n}$ denote the Fourier transform of $f_{n}$. As remarked by Paley and Wiener [2], since the Fourier transform is normpreserving in $L_{2}(R)$, the sequences $\left\{f_{n}\right\}$ and $\left\{F_{n}\right\}$ have the same closure properties. Since $F_{n}(x)=F(-x) \cdot \exp \left(-c_{n} x i\right)$, making the change of variable $x \rightarrow-x$, we obtain the following, fully equivalent proposition:

THEOREM 3. Let $F$ be a function in $L_{2}(R)$, such that $F(x) \neq 0$ for all real $x$, and let $F_{n}$ be defined by

$$
F_{n}(x)=F(x) \cdot \exp \left(c_{n} x i\right) .
$$

Then, if $F$ satisfies condition (i) of Theorem 2, the divergence of $S(\varepsilon)$ for some $\varepsilon>0$ suffices for the sequence $\left\{F_{n}\right\}$ to span $L_{2}(R)$, whereas if $F$ satisfies condition (ii) thereof, the divergence of $S(0)$ is necessary for this sequence to span $L_{2}(R)$.

In particular, we have

COROLlaRy 2. If $S(\varepsilon)$ is divergent for some $\varepsilon>0$, then the sequence $\left\{\exp \left(c_{n} x i-x^{2}\right)\right\}$ spans $L_{2}(R)$. Conversely, if this sequence spans $L_{2}(R)$, then $S(0)$ must be divergent.

\footnotetext{
'ACKNowledgement. The author is grateful to Dr. A. Atzmon for suggesting the present, more elegant, statement of conditions (i) and (ii) of Theorem 2.
} 
This corollary should be compared with the standard density theorems for sequences of the form $\left\{\exp \left(c_{n} t i\right)\right\}$ on finite intervals (see $\mathrm{N}$. Levinson [3, Chapters 1 and 2], and L. Schwartz [4, Chapter 3]).

An interesting feature of Theorems 2 and 3 is that they seem to be the first density theorems that do not deal with sequences of functions generated in some way by entire functions of exponential type, and are therefore proved without recourse to Paley and Wiener's classical theorem on the representation of such functions as Fourier transforms of functions having bounded support. Note, moreover, that Theorems 2 and 3 apply to classes of sequences of functions much larger in size than, for example, those to which the Müntz-Sźasz theorem applies. The function $\exp \left(-t^{2}\right)$ is just one of a large class of functions satisfying the conditions of Theorem 2. Specifically, we have

LEMMA. Let $f$ be a function in $L_{2}(R)$ such that $1 / F$ is an entire function of order 2 , and the set of zeros of $1 / F$ is bounded away from the real axis and has an exponent of convergence smaller than 2. Then the function $f$ satisfies conditions (i) and (ii) of Theorem 2.

The conditions of the preceding Lemma are satisfied by a large set of "Pólya frequency functions". An account of the theory of these functions can be found in the monograph of Karlin [5] (see, in particular, Theorem 3.2 on p. 345). For an account of their connection with the convolution transform and the heat equation, see Hirschman and Widder [6], and also the work of D. Leviatan et al. [7], [8]. The inversion theorems developed in the three preceding references do not seem directly applicable to the proof of our results.

It should be remarked that the condition that the function $1 / F$ should be of order 2 cannot be changed with impunity. This is illustrated by the following

ExAMPLE. Let $f(t)=\exp (1-t)$ if $t \geqslant 1$, and $f(t)=0$ if $t<1$. Then $1 / F(z)=(i z+1) \exp (i z)$ is an entire function of order 1 . However, no sequence of the form $\left\{f\left(c_{n}-t\right)\right\}$ may span $L_{2}(R)$. Indeed, if $g$ is a linear combination of functions of the form $f\left(c_{n}-t\right)$, it admits of a representation of the form $g(t)=k(t) \exp (-t)$, where $k(t)$ is constant in each interval $\left(c_{n}, c_{n+1}\right)$. This implies that $\left\{f\left(c_{k}-t\right)\right\}$ cannot span even $L_{2}\left(c_{n}, c_{n+1}\right)$. (Incidentally, the function $f$ thus defined is a Pólya frequency function (cf. $[5, \mathrm{p}$. 333]).)

The preceding example is due to $Z$. Ziegler and J. Tzimbalario.

Theorems 2 and 3 obtain for an unbounded interval. For the case of a bounded interval we can prove, with the aid of standard techniques, the following: 
THEOREM 4. Let $(a, b)$ be $a$ bounded interval and let $f(t)=\exp \left(-c^{2} t^{2}\right)$, $c \neq 0$. Then $\left\{f\left(c_{n}-t\right)\right\}$ spans $L_{2}(a, b)$ if, and only if, the series $\Sigma^{\prime}\left|c_{n}\right|^{-1}$ diverges.

Proof of Theorem 4. Sufficiency: Let $q$ belong to $L_{2}(a, b)$, and assume that

$$
\int_{a}^{b} \exp \left[-c^{2}\left(c_{n}-t\right)^{2}\right] q(t)=0, \quad n=0,1,2, \ldots
$$

Let $d=1 / c$; making a change of variable of the form $t \rightarrow d t+a$, and defining $p(t)=q(d t+a), l=c(b-a)$ and $d_{n}=c\left(c_{n}-a\right)$, we see that (2) reduces to

$$
\int_{0}^{l} \exp \left[-\left(d_{n}-t\right)^{2}\right] p(t) d t=0, \quad n=0,1,2, \ldots,
$$

which is equivalent to

$$
\int_{0}^{l} \exp \left[-\left(2 d_{n} t-t^{2}\right)\right] p(t) d t=0, \quad n=0,1,2, \ldots
$$

However, $\exp \left(-t^{2}\right) p(t)$ belongs to $L_{2}(0, l)$, and the conclusion follows from the Müntz-Szász theorem by a change of variable of the form $\exp (-t) \rightarrow t$ (cf. for example [2, pp. 30-36]).

Necessity. Assume the series $\Sigma^{\prime}\left|c_{n}\right|^{-1}$ converges, and define $w_{n}=-2 c c_{n} i$. From a theorem of Luxemburg and Korevaar [9, Theorem 5.2], we know there exists a function $G(z)$, not identically zero, that vanishes at the points $w_{n}$ and admits of a representation of the form

$$
G(z)=\int_{a}^{b} \exp (-i z t) g(t) d t,
$$

where $g$ is in $L_{2}(a, b)$. Defining $q(t)=g(t) \exp \left(c^{2} t^{2}\right)$, this means that

$$
\int_{a}^{b} \exp \left(-2 c c_{n} t-c^{2} t^{2}\right) q(t) d t=0, \quad n=0,1,2, \ldots
$$

Therefore, $q$ satisfies (2) without being identically zero. Since $q$ is clearly in $L_{2}(a, b)$, we thus see that $\left\{f\left(c_{n}-t\right)\right\}$ does not span $L_{2}(a, b)$, and the conclusion follows. Q.E.D.

Proof of Theorem 1. Assume there is a function $p$ in $L_{2}(a, b)$ such that

$$
\int_{a}^{b} h\left(c_{n}-t\right) p(t) d t=0, \quad n=0,1,2, \ldots
$$

Extend $p$ to the whole of $R$ by stipulating it to vanish outside $(a, b)$. Defining $q=h * p$, (3) simply means that

$$
q\left(c_{n}\right)=0, \quad n=0,1,2, \ldots
$$

Let $k=g * p$; since $g$ is in $L_{1}(R)$ and $p$ belongs to $L_{2}(R), k$ is in $L_{2}(R)$, and 
it is clear that $q=(f * g) * p=f * k$. A straightforward computation shows that $k$ must vanish outside $(2 a, 2 b)$. Thus,

$$
q(x)=\int_{2 a}^{2 b} f(x-t) k(t) d t
$$

Since $\left\{f\left(c_{n}-t\right)\right\}$ spans $L_{2}(2 a, 2 b)$, we conclude from (4) that $k$ vanishes almost everywhere in $R$. Thus, $0=K=G P$ almost everywhere in $R$. By hypothesis $G$ is different from zero a.e. in $R$. Thus $P=0$ a.e. in $R$, and therefore also $p$. Q.E.D.

REMARK. The preceding proof is similar to one of the proofs of Wiener's theorem (cf. Katznelson [10, p. 145, Exercise 7]).

Proof OF TheOREM 3. We shall use the notation of Boas [11].

Sufficiency. We shall first show that the assertion holds for all functions of the form $F(x)=\exp \left(-d^{2} x^{2}\right)$, where $d$ is any nonzero real number. This is equivalent to proving the sufficiency part of Theorem 2 for all functions of the form $f(t)=\exp \left(-a^{2} t^{2}\right)$, where $a$ is any nonzero real number (for if $F$ is the Fourier transform of $f$, then $\left.d^{2}=1 /\left(4 a^{2}\right)\right)$.

Assume therefore that there is a function $p$ in $L_{2}(R)$, not almost everywhere zero, such that

$$
\int_{R} \exp \left[-a^{2}\left(c_{n}-t\right)^{2}\right] p(t) d t=0, \quad n=0,1,2, \ldots
$$

Without loss of generality, we can assume that

$$
\int_{R}|p(t)|^{2} d t=|a|(2 / \pi)^{1 / 2}
$$

The proof will be achieved once we show that the series $S(\varepsilon)$ is convergent for all $\varepsilon>0$. Define

$$
h(z)=\exp \left(a^{2} z^{2}\right) \int_{R} \exp \left[-a^{2}(z-t)^{2}\right] p(t) d t .
$$

Clearly $h\left(c_{n}\right)=0, n=0,1,2, \ldots$, and

$$
h(z)=\int_{R} \exp \left[2 a^{2} t z-a^{2} t^{2}\right] p(t) d t .
$$

It is clear that $h(z)$ is an entire function (cf. Widder [12, p. 57]). Moreover, $h$ cannot be identically zero, for $\exp \left(-a^{2} t^{2}\right) h(t)=\exp \left(-a^{2} t^{2}\right) * p(t)$; thus, were $h$ to vanish identically, we would conclude that the Fourier transform of $p$ vanishes a.e., and thus also $p$, which is a contradiction.

Applying Hölder's inequality, we see that, if $z=x+y i$,

$$
|h(z)|^{2} \leqslant|a|\left(\frac{2}{\pi}\right)^{1 / 2} \int_{R} \exp \left[4 a^{2} t x-2 a^{2} t^{2}\right] d t=\exp \left(2 a^{2} x^{2}\right) .
$$

We have therefore shown that $h$ is an entire function of order at most 2. Thus, applying [11, 2.5.19], the conclusion follows. To complete the proof, let $F$ 
satisfy condition (i), and assume that $S(\varepsilon)=\infty$ for some $\varepsilon>0$. Assume $m$ is a function in $L_{2}(R)$ such that

$$
\int_{R} \exp \left(c_{n} x i\right) F(x) m(x) d x=0, \quad n=0,1,2, \ldots
$$

Setting $G(x)=\exp \left[\frac{1}{2} a^{2} x^{2}\right] F(x) m(x)$, the preceding equation can be written as

$$
\int_{R^{\prime}} \exp \left(c_{n} x i\right) \exp \left(-\frac{1}{2} a^{2} x^{2}\right) G(x) d x=0, \quad n=0,1,2, \ldots
$$

Since condition (i) implies that $G$ is obtained through multiplication of $m$ by a bounded function, and $m$ was assumed to be in $L_{2}(R)$, it is clear that also $G$ is in $L_{2}(R)$. Since we have shown that the sequence $\left\{\exp \left(c_{n} x i-\frac{1}{2} a^{2} x^{2}\right)\right\}$ spans $L_{2}(R)$, it is clear that $G=0$ a.e. Since, by hypothesis, $F$ never vanishes, this implies that $m=0$ a.e., and the conclusion follows.

Necessity. Clearly $\left\{c_{n}\right\}$ must be an infinite sequence. Making, if necessary, a change of variable of the form $t \rightarrow t+t_{0}$ we can assume, without loss of generality, that all the numbers $c_{n}$ are different from zero. Assume that $S(0)$ converges, and let $q_{1}(z)=\Pi E\left(-z / c_{n}, 1\right)$ and $q(z)=q(\mu, z)=$ $\exp \left(-\frac{1}{2} \mu z^{2}\right) q_{1}(z)$, where $\mu>0$. It is clear that $q$ is an entire function. We shall now follow a procedure inspired by the proof of $[13$, Chapter 1 , Theorem 2]. From [11, 2.10.13] we know that $q_{1}$ is of growth $(2,0)$. It is therefore easy to see that the following inequalities obtain:

$$
q(z)=O[\exp (-\alpha|x|)], \quad|x| \rightarrow \infty,
$$

on any strip of the form $|y|<\delta$, and for every real number $\alpha$.

$$
\int_{R}|q(x+y i)|^{2} d x \leqslant c_{1}^{2} \exp \left(\mu y^{2}\right)
$$

for every real number $y$, and

$$
\int_{R}|(x+y i) q(x+y i)|^{2} d x \leqslant c_{2}^{2} \exp \left(\mu y^{2}\right)
$$

for every real number $y$. Note that also $i z q(z)$ satisfies a condition like (5). Thus, applying a theorem of Titchmarsh [14, p. 44, Theorem 1.27] to both $q(z)$ and $i z q(z)$, it is easily seen that there is an entire function $h=h(\mu, \cdot)$, such that both $h(t)$ and $h^{\prime}(t)$ are in $L_{2}(R), q(z)$ is the Fourier transform of $h$, and $i z q(z)$ is the Fourier transform of $h^{\prime}$.

Let $t>0$, and $I=[t, t+1]$. Applying Parseval's identity we see that, for all real $y$,

$$
\begin{aligned}
\int_{I}|h(s)|^{2} \exp (2 s y) d s & <\int_{R}|h(s)|^{2} \exp (2 s y) d s \\
& =\int_{R}|q(x+y i)|^{2} d x \leqslant c_{1}^{2} \exp \left(\mu y^{2}\right) .
\end{aligned}
$$


Since $s \geqslant t$ on $I$, by setting $y=t / \mu$, we obtain

$$
\exp \left(\frac{2 t^{2}}{\mu}\right) \int_{I}|h(s)|^{2} d s<c_{1}^{2} \exp \left(\frac{t^{2}}{\mu}\right) .
$$

Thus,

$$
\int_{I}|h(s)|^{2} d s<c_{1}^{2} \exp \left(-\frac{t^{2}}{\mu}\right),
$$

and from (7) we similarly see that

$$
\int_{I}\left|h^{\prime}(s)\right|^{2} d s<c_{2}^{2} \exp \left(-\frac{t^{2}}{\mu}\right) .
$$

In view of (8), there must be a point $t_{0}$ in $I$ where $h$ satisfies the inequality

$$
\left|h\left(t_{0}\right)\right|<c_{1} \exp \left(-\frac{t^{2}}{2 \mu}\right) .
$$

Hence, since $t<t_{0}$, applying the Cauchy-Schwarz inequality and (9) we see that

$$
\begin{aligned}
|h(t)| & =\left|h\left(t_{0}\right)+\int_{t_{0}}^{t} h^{\prime}(s) d s\right|\left\langle\left|h\left(t_{0}\right)\right|+\int_{t}^{t_{0}} 1 \cdot\left|h^{\prime}(s)\right| d s\right. \\
& <\left|h\left(t_{0}\right)\right|+\left|t_{0}-t\right|^{1 / 2}\left[\int_{t}^{t_{0}}\left|h^{\prime}(s)\right|^{2} d s\right]^{1 / 2} \\
& <\left|h\left(t_{0}\right)\right|+\left[\int_{I}\left|h^{\prime}(s)\right|^{2} d s\right]^{1 / 2} \\
& <c_{1} \exp \left(-\frac{t^{2}}{2 \mu}\right)+c_{2} \exp \left(-\frac{t^{2}}{2 \mu}\right) \\
& =c \exp \left(-\frac{t^{2}}{2 \mu}\right),
\end{aligned}
$$

where $c=c_{1}+c_{2}$. Thus, if $t>0$, we have shown that

$$
|h(t)|<c \exp \left(-\frac{t^{2}}{2 \mu}\right)
$$

Since (8) and (9) hold also for the function $h(-t)$ (for its Fourier transform is $q(-z)$ ), we readily see that (10) holds for every real value of $t$.

Referring now to condition (ii) of Theorem 2, we know from (10) that there exists a number $\mu_{0}$, such that

$$
h\left(\mu_{0}, t\right)=O\left[\exp \left(-b^{2} t^{2}\right)\right], \quad|t| \rightarrow \infty .
$$

It is therefore clear that the function $m(t)$ defined by 
is in $L_{2}(R)$. However,

$$
m(t)=h\left(\mu_{0}, t\right) / F(t)
$$

$$
(2 \pi)^{1 / 2} q\left(\mu_{0}, z\right)=\int_{R} \exp (-z t i) F(t) m(t) d t .
$$

Since $q\left(\mu_{0},-c_{n}\right)=0$ for $n=0,1,2, \ldots$, and $m$ is clearly not identically zero, we have therefore shown that the sequence $\left\{F(t) \exp \left(c_{n} t i\right)\right\}$ does not span $L_{2}(R)$. Q.E.D.

Proof of LeMma. From Hadamard's factorization theorem we know that

$$
1 / F(z)=K \exp \left(b_{1} z^{2}+b_{2} z\right) E(z),
$$

where $E(z)$ is the canonical product of the zeros of $1 / F$. Without any loss of generality we can assume that $K=1$, and that $b_{1}$ and $b_{2}$ are real numbers. Indeed, the assumption that $b_{1}$ and $b_{2}$ are complex will only introduce a weight function whose absolute value equals one for any real value of the argument $z$. Let $k$ be the exponent of convergence of the zeros of $1 / F$. Since $E(z)$ is of order $k$ (cf. $[11,2.6 .5]$ ), and $k$ is less than 2 by hypothesis, the proof of condition (ii) readily follows. To prove condition (i), choose some number $r$ from the interval $(k, 2)$, and note that for $|z|$ large enough,

$$
|E(z)| \leqslant \exp |z|^{r}
$$

We thus conclude from (11) that for $|x|$ large enough,

$$
|F(x)| \geqslant \exp \left(-b_{1} x^{2}-b_{2} x-|x|^{r}\right)
$$

Since $F(x)$ must clearly be in $L_{2}(R)$ (for it is the Fourier transform of a function in $L_{2}(R)$ ), we conclude from the preceding inequality that $b_{1}$ must be nonnegative. Since $1 / F$ is a function of order 2 , it is also clear from (12) that $b_{1}$ cannot equal zero. We have thus proved that $b_{1}$ is strictly positive. Now, $E$ is a canonical product of order $k$; thus, if $s$ is any positive real number, we know from $[11,2.6 .18]$ that

$$
\log |E(z)| \geqslant-|z|^{r}
$$

provided that $|z|$ is large enough and $z$ is outside the circles of center $z_{n}$ and radius $\left|z_{n}\right|^{-s},\left|z_{n}\right|>1$ (where $\left\{z_{n}\right\}$ is the set of zeros of $1 / F$ ). Since $\left\{z_{n}\right\}$ cannot have points of accumulation, it is readily seen that there exists a number $m>1$, such that for every point $z_{n}$, if its absolute value is larger than 1 , it is also larger than $m$. Thus, by taking $s$ to be sufficiently large, we can make the supremum of the radii of the circles under consideration arbitrarily small. Since by hypothesis the set $\left\{z_{n}\right\}$ is bounded away from the real axis, we readily conclude that (13) holds for all points in some strip of the form 
$|y|<\delta$ (where $z=x+y i$ ), provided they are sufficiently distant from the origin, and the conclusion readily follows from (11), (13), and the positivity of $b_{1}$. Q.E.D.

REMARKS. The preceding results give rise to several questions. The first one is whether a theorem similar to Theorem 2 is valid on $L_{p}(R)$, for some value of $p$ other than 2. Our method of proof is applicable only in $L_{2}(R)$, for we have made essential use of a duality argument to obtain Theorem 2 from Theorem 3. Another question is the following: in Theorem 2 we assumed that, roughly speaking, $F(x)$ behaves like $\exp \left(-x^{2}\right)$. The question thus naturally arises as to what happens if $F(x)$ behaves like $\exp \left(-|x|^{p}\right)$, for some $p$ other than 2 . This problem is at present open. It would also be interesting to study these problems in the interval $(0, \infty)$, where the theory of quasianalytic functions seems to have some application.

As a final remark, we would like to point out that other theorems concerning the closure of sequences of translates have been considered by $R$. E. Edwards in his doctoral dissertation (see [15]), and subsequent papers (see [16] and references thereof), and by M. M. Džrbašjan and V. M. Martirosjan [17].

\section{REFERENCES}

1. N. Wiener, The Fourier integral and certain of its applications, Cambridge Univ. Press, Cambridge, 1933; reprint, Dover, New York, 1958.

2. R. E. A. C. Paley and N. Wiener, Fourier transforms in the complex domain, Amer. Math. Soc. Colloq. Publ., no. 19, Amer. Math. Soc., Providence, R. I., 1934.

3. N. Levinson, Gap and density theorems, Amer. Math. Soc. Colloq. Publ., no. 26, Amer. Math. Soc., Providence, R. I., 1940.

4. L. Schwartz, Etude des sommes d'exponentielles, 2nd ed., Hermann, Paris, 1959.

5. S. Karlin, Total positivity, Stanford Univ. Press, Stanford, Calif., 1968.

6. I. I. Hirschman and D. V. Widder, The convolution transform, Princeton Univ. Press, Princeton, N. J., 1955.

7. D. Leviatan, $A$ new approach to the representation theory for convolution transforms, Pacific J. Math. 35 (1970), 441-449.

8. C. Danon and D. Leviatan, $A$ representation theorem for convolution transform with determining function in $L^{p}$, Pacific J. Math. 62 (1976), 81-86.

9. W. A. J. Luxemburg and J. Korevaar, Entire functions and Müntz-Szász type approximation, Trans. Amer. Math. Soc. 157 (1971), 23-37.

10. Y. Katznelson, An introduction to harmonic analysis, Wiley, New York, 1968.

11. R. P. Boas, Jr., Entire functions, Academic Press, New York, 1954.

12. D. V. Widder, The Laplace transform, Princeton Univ. Press, Princeton, N. J., 1972.

13. I. M. Gel'fand and G. E. Silov, Fourier transforms of rapidly increasing functions and questions of the uniqueness of the solution of Cauchy's problem, Uspehi Mat. Nauk 8 (1953), 3-54; English transl., Amer. Math. Soc. Transl. (2) 5 (1957), 221-274.

14. E. C. Titchmarsh, Theory of Fourier integrals, 2nd ed., Oxford Univ. Press, London, 1948; reprinted 1967.

15. R. E. Edwards, The translations of a function holomorphic in a half-plane and related problems in the real domain, Proc. London Math. Soc. (3) 1 (1951), 118-128.

16. Functional analysis, theory and applications, Holt, Rinehart and Winston, New York, 1965. 
17. M. M. Džrbašjan and V. M. Martirosjan, Theorems of Paley-Wiener and Müntz-Szász type, Dokl. Akad. Nauk SSSR 225 (1975), 1001-1004 = Soviet Math. Dokl. 16 (1975), 1559-1563 (1976).

Departmant of Mathematics, Ben Gurion University of the Negev, BeERsheba, Israel

Current address: Department of Mathematics, Auburn University, Auburn, Alabama 36830 Island 02881 